\title{
A Comparative Study on Position Fixing with GPS to Ascertain Positional Accuracy*
}

\author{
C. B. Boye and I. Yakubu
}

Boye, C. B. and Yakubu, I., (2010), "A Comparative Study on Position Fixing with GPS to Ascertain Positional Accuracy", Ghana Mining Journal, Vol. 12, pp. 1 - 6.

\begin{abstract}
Various techniques have been employed to determine the relative or absolute positions of locations on the surface of the earth to promote effective land management. Continuous Observation Reference Station (CORS) is one of the latest techniques employed in differential GPS positioning. In order to ascertain the positional accuracy of a newly established CORS equipment located on the campus of the University of Mines and Technology (UMaT), this comparative study was carried out. Selected positions around the campus were surveyed and their coordinates computed using a temporarily occupied base and then a CORS base, and the results were compared with the coordinates obtained from a conventional precise traverse. It was observed that the Eastings coordinates obtained from the temporarily occupied reference station compares favourably with those of the conventional method and the CORS with variations in the second and third decimals. Standard errors of $-0.144 \mathrm{~m}$ and $0.806 \mathrm{~m}$ respectively were obtained for the Northings and elevations respectively between the CORS coordinates and that of the conventional method. It was observed that there is a strong correlation between the coordinates of the control points and the relation between the Eastings, Northings and heights were all linear. It could be concluded that the CORS station, which is under study, could be used with minimal error for planimetric and or cadastral surveying purposes.
\end{abstract}

\section{Introduction}

The techniques employed in the determination of positions of points on the surface of the earth have seen tremendous improvements over the decades. These are the traditional methods and the use of Global Positioning System (GPS) techniques. The traditional methods of surveying include triangulation, traversing and leveling which were used extensively before the 1980 s prior to the advent of GPS surveying (Anon, 2008). In the conventional methods of surveying inter-visibility is required and calls for clearing to achieve the necessary lineof-sight. GPS surveys, on the other hand, utilise a number of satellites as the space references to compute the coordinates of locations where GPS receivers are mounted, and has the advantage of speed, cost effectiveness, fixes positions accurately with greater flexibility and has accessibility to remote areas. Some techniques are employed to further improve the accuracy of GPS observed positions. Some of these methods include Static GPS surveying, differential GPS, Real Time Kinematics (RTK) and lately the use of CORS. Static surveying involves occupying a point and collecting satellite telemetry for a period (usually 2 hours, time adjusted by distance to reference station). GPS positioning method uses relative positioning techniques where a control in the vicinity is incor- porated in the computation of the position to significantly reduce the errors that occur in static or absolute positioning (Torge, 2001). RTK surveying is where GPS signal corrections are transmitted in real time from a reference receiver at a known location to one or more remote rover receivers. CORS is a GPS receiver located permanently at a strategic position with very accurately predetermined coordinates, and equipped with downloading facilities which process the coordinates of the station and makes them accessible to users on the internet (Anon, 2004). CORS with RTK technique offers surveying on the fly, without loss of accuracy, and is supported by base stations with internet Transmission Control Protocol (Anon, 2009).

The National Geodetic Survey (NGS) of Michigan, USA for example collects the data from their CORS GPS receivers and makes the data available to GPS surveyors so that they can correct for any errors that are in the GPS signals (Anon, 2004). On -line Positioning service software packages have been provided in some countries for GPS users to enable easier ways of determining positions with respect to the CORS network. The University of Mines and Technology (UMaT), Tarkwa, received a donation of a CORS and its accessories to serve the University community, practicing surveyors and the allied mining industry within $40 \mathrm{~km}$ radius.

\footnotetext{
* Manuscript received May 17, 2010

Revised version accepted October 2, 2010
} 
The UMaT CORS, like others, was mounted with the aim of providing high order GPS reference data that will help establish controls with greater accuracy and flexibility while performing surveying for a variety of applications for example. In the provision of reliable and consistent data for establishing spatial relations, legal boundaries, infrastructure projects, mining related projects, environmental assessment and private enterprise development. The accuracy of the base used in postprocessing of any fixed position, to a large extent, affect the correctness of the resulting computed coordinates. To ascertain the accuracy of DGPS surveys tied to a permanent reference station compared to those tied to the CORS is of primary concern more especially to cadastral surveyors where discrepancies in fixed boundary coordinates may degenerate into legal boundary conflicts leading to the possible loss of property and lives.

In this paper, the results of a study that was undertaken to determine the accuracy of GPS positioning using the temporary occupied reference station and the CORS as respective bases are given. The results are compared with those of the conventional method.

\section{Study Area}

The area of study covers part of the UMaT Campus and the Tarkwa Township. Tarkwa is the capital of the Tarkwa Nsuaem Municipal Assembly, a mining community in the Western Region of Ghana. The town is about $85 \mathrm{~km}$ from Takoradi, which is the regional capital, $233 \mathrm{~km}$ from Kumasi and about $317 \mathrm{~km}$ from Accra (Kesse, 1985). The University campus covers an area of approximately $1.39 \mathrm{~km}^{2}$ of undulating land and pleasant surroundings, about $2 \mathrm{~km}$ south of Tarkwa. Already established survey controls stations used in the study area are shown in Fig. 1.

\section{Materials and Methods}

The materials and methods employed in this research are discussed in the following sections.

\subsection{Materials}

Control stations for the research were identified and primary data collected by field survey using GPS receivers. Data obtained was processed using spectrum GPS software and analysed with Microsoft Excel.

\subsubsection{Equipment}

The field equipment includes four single frequency GPS receivers, one CORS and accessories. Also a total station and two reflector targets were used to determine the coordinates of the selected points using classical surveying techniques.

\subsubsection{Software}

The Spectrum GPS software Version 3.3 was used for the post-processing of the GPS data, while Microsoft Excel and ArcGIS 9.2 were used for the regression analysis and the plotting of the points respectively.

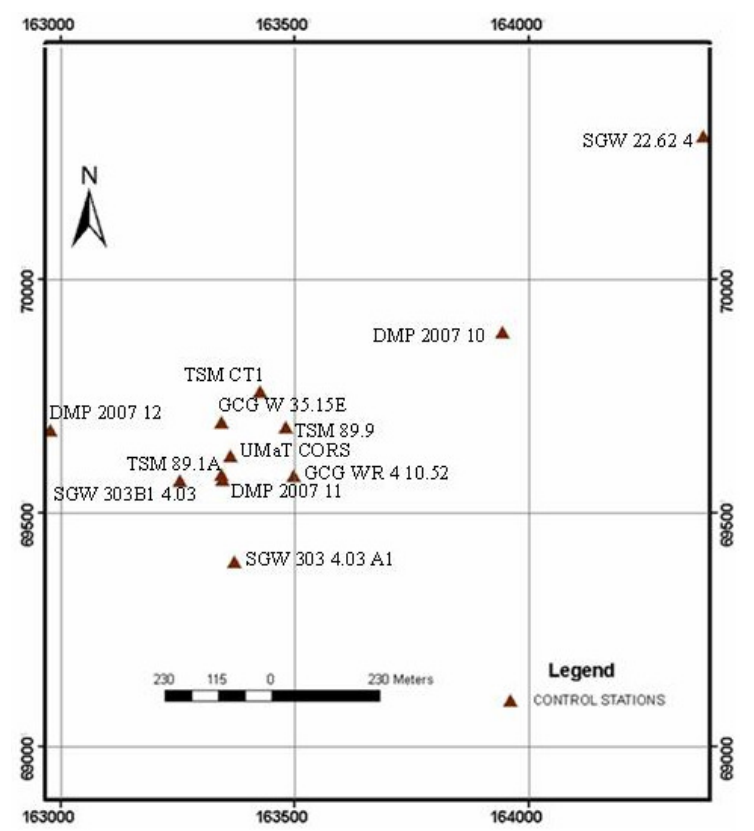

Fig. 1 Sample Location of Control Stations

\subsection{Methods}

A DGPS survey was conducted with the CORS and a temporary occupied station used as respective bases. A conventional survey was also conducted.

\subsubsection{Sampling}

A reconnaissance survey was conducted during which the site used for the work was selected. The conditions of the instruments used were also ascertained by checking the battery strength. The receivers were also used to pick at random points and the information downloaded and processed to see if the receivers were functioning properly by comparing the coordinates obtained with the actual coordinates of those points. The discrepancies were found to be minimal and therefore the receivers were considered to be in a good working condition. Also a point of departure test was conducted as a check on the stability of the base control point used, and was found to be good. Eleven controls were selected for the survey such that there was clear view of the sky (see Fig. 1). 


\subsubsection{Using the CORS}

In control point positioning, the coordinates of the antenna position at an unknown point were found with respect to the WGS84 reference frame (local reference frame). In this method, the reference position is occupied and the unknown position is in turn occupied, with the known positions of the satellites, the position of the unknown is computed using a similar method to that of resection. The static technique was used and the rover positions were obtained by the 'leap frog' method while the CORS was operational. The rover receivers were set up on the points whose coordinates were required. The rover was switched off when a link between the rover and the base station was created. The occupation time was between 20 and 40 minutes since the distance between the base and the rover stations was less than $2 \mathrm{~km}$. The time the rover was switched off was recorded. This procedure was repeated until all the controls were occupied. In processing, the downloaded coordinates of the CORS were also used as a base in postprocessing of the coordinates of the unknown control points.

\subsubsection{Using the Temporary Occupied Refer- ence Station}

With the temporary occupied reference station technique, a GPS receiver is mounted on a control point whose coordinates are known and serves as the reference station of the survey. That receiver is referred to as the base receiver of the survey which had the description SG W 22/64 4 and remains until the entire survey is completed. The rest of the receivers (which are referred to as the roving receivers), are moved around the points whose coordinates are required. The time the receiver was switched on; the receiver's serial number and the name of the occupied pillar were recorded in the field book. The roving receivers were also set up on the points whose coordinates were required. The rover was switched off and the time was recorded when a link between the rover and the base station was created. The occupation time was between 30 and 40 minutes. This procedure was repeated until all the controls were occupied. The base receiver was switched off and the time recorded. In processing the data to obtain the coordinates of the points, the coordinates of the reference station were used to compute the differential which in turn was used to adjust the GPS coordinates of the new points.

\subsubsection{Using the Classical Survey Method}

To enable effective comparison of the two techniques, a classical survey method was carried out to determine the coordinates of the selected controls. A close link traverse was conducted over the sampled controls using a total station; an observation was also made to the mast of the CORS. The closing error between the starting and the closing coordinates by manual computation averaged $0.003 \mathrm{~m}$.

\section{Results}

Results obtained from processing the data using both the temporary occupied reference station and the CORS as a base were computed. These were compared with the coordinates of the points determined by running a precise traverse over the controls. Tables 1 and 2 show the coordinates that were obtained and their respective discrepancies from the Conventional method's coordinates.

The first row of Table 1 shows that sample station TSM CT1 has GPS post-process average coordinates of $163427.194 \mathrm{~m}$ in the eastings, 69760.065 $\mathrm{m}$ in the northings and $81.719 \mathrm{~m}$ in elevation with respect to the CORS; $163427.178 \mathrm{~m}, 69760.245 \mathrm{~m}$ and $80.846 \mathrm{~m}$ in the eastings, northings and elevation respectively with respect to the temporary occupied reference station; and $163427.176 \mathrm{~m}$, $69760.217 \mathrm{~m}$ and $80.834 \mathrm{~m}$ in the eastings, northings and elevation respectively by the traditional method. This gives differences of $0.038 \mathrm{~m}$ in the eastings, $-0.144 \mathrm{~m}$ in the northings and $0.806 \mathrm{~m}$ in the elevation between the CORS coordinates and those of the temporary occupied reference station coordinates. Also there were discrepancies of $0.058 \mathrm{~m}, 0.060 \mathrm{~m}$ and $0.076 \mathrm{~m}$ in the eastings, northings and elevation respectively between the coordinates of the temporary occupied reference station and those of the traditional method. In addition, there were differences of $0.038 \mathrm{~m},-0.110 \mathrm{~m}$ and $0.878 \mathrm{~m}$ in the eastings, northings and elevation respectively between the CORS coordinates and those of the Conventional method coordinates (Table 2).

\subsection{Analysis of Results}

Linear regression analysis was applied to the scatter diagrams of the coordinates to assess the relationships between the coordinates of the Conventional method, the temporary occupied reference station coordinates, and the CORS coordinates. Figs. 2 to 4 show the relationships established between the variables (eastings, northings and elevations).

The post-processed coordinates of the eastings, nothings and heights of the coordinates of the Conventional method against those of CORS show a strong correlation with a coefficient of deterministic $\left(\mathrm{R}^{2}\right)$ of 1 in the eastings, northings and heights. Figs. 5 to 7 show the eastings, nothings and elevations of the coordinates of the Conventional method plotted against those of the temporary oc- 
cupied reference post-processed coordinates. In the same way, the post-processed coordinates of the eastings, nothings and heights of the coordinates of the Conventional method against those of temporary occupied reference station have a strong correlation with a coefficient of deterministic $\left(\mathrm{R}^{2}\right)$ of 1 in the eastings, northings and heights.

\section{Discussion}

From the analysis of the results obtained using the three methods, it was observed that variations between the data post-processed using the temporarily occupied reference station and the CORS were very small. Discrepancies in the eastings and northerns coordinates, and elevation were determined with respect to the coordinates of the Conventional method of the same control points (see Table 2).

The coordinates obtained from the CORS, temporarily occupied reference station and the conventional method compare favourably with each other with variations in the second and third decimals. From Table 2 there were, however, small variations of averaging values of $0.038 \mathrm{~m},-0.144 \mathrm{~m}$ and $0.806 \mathrm{~m}$ for the eastings, nothings and heights respectively for the CORS and temporarily occupied reference station.

Table 1 Coordinates of Sample Points using CORS, Temporary Occupied Referenced Station and by the Conventional Method

\begin{tabular}{|c|c|c|c|c|c|c|c|c|c|}
\hline Points Description & \multicolumn{3}{|c|}{ CORS } & \multicolumn{3}{|c|}{$\begin{array}{c}\text { Temporary Occupied Reference Station } \\
\text { (TOR) }\end{array}$} & \multicolumn{3}{|c|}{ Conventional Method (CM) } \\
\hline TSM CT1 & 163427.194 & 69760.065 & 81.719 & 163427.178 & 69760.245 & 80.846 & 163427.176 & 69760.217 & 80.834 \\
\hline SGW $3034.03 \mathrm{~A} 1$ & 163372.166 & 69396.262 & 68.264 & 163372.201 & 69396.405 & 67.415 & 163372.252 & 69396.403 & 67.342 \\
\hline GCG WR 410.52 & 163499.247 & 69581.511 & 72.991 & 163499.239 & 69581.512 & 72.26 & 163499.265 & 69581.574 & 72.214 \\
\hline TSM 89.1A & 163344.962 & 69582.352 & 75.786 & 163344.988 & 69582.513 & 74.939 & 163345.017 & 69582.572 & 74.846 \\
\hline DMP 200712 & 162979.339 & 69678.616 & 99.625 & 162979.351 & 69678.764 & 98.915 & 162979.338 & 69678.749 & 98.733 \\
\hline TSM 89.9 & 163482.643 & 69684.168 & 72.875 & 163482.946 & 69684.312 & 72.055 & 163482.625 & 69684.311 & 71.982 \\
\hline DMP 200711 & 163346.75 & 69571.345 & 75.647 & 163346.762 & 69571.505 & 74.788 & 163346.834 & 69571.117 & 74.683 \\
\hline UMaT CORS & 163364.573 & 69622.35 & 88.064 & & & & & & \\
\hline
\end{tabular}

Table 1 Relative Discrepancies between the Coordinates of CORS, the Temporary Occupied Reference Station, and the Conventional Method

\begin{tabular}{|c|c|c|c|c|c|c|c|c|c|}
\hline Points Description & \multicolumn{3}{|c|}{ Discrepancies (CORSTOR) } & \multicolumn{3}{|c|}{ Discrepancies (TORTM) } & \multicolumn{3}{|c|}{ Discrepancies (CORSTM) } \\
\hline TSM CT1 & 0.016 & -0.180 & 0.873 & 0.002 & 0.028 & 0.012 & 0.018 & -0.152 & 0.885 \\
\hline SGW $3034.03 \mathrm{~A} 1$ & 0.035 & -0.143 & 0.849 & 0.051 & 0.002 & 0.073 & 0.086 & -0.141 & 0.922 \\
\hline GCG WR 410.52 & 0.008 & -0.001 & 0.731 & 0.026 & 0.062 & 0.046 & 0.018 & -0.063 & 0.777 \\
\hline SGW 22.624 & 0.002 & -0.164 & 0.740 & 0.000 & 0.000 & 0.000 & 0.002 & -0.164 & 0.740 \\
\hline TSM 89.1A & 0.026 & -0.161 & 0.847 & 0.029 & 0.059 & 0.093 & 0.055 & -0.22 & 0.940 \\
\hline DMP 200712 & 0.012 & -0.148 & 0.710 & 0.013 & 0.015 & 0.182 & 0.001 & -0.133 & 0.892 \\
\hline TSM 89.9 & 0.303 & -0.144 & 0.820 & 0.321 & 0.001 & 0.073 & 0.018 & -0.143 & 0.893 \\
\hline GCG W 35.15E & 0.005 & -0.159 & 0.825 & 0.115 & 0.075 & 0.033 & 0.120 & -0.084 & 0.858 \\
\hline UMaT CORS & 0.016 & -0.138 & 0.875 & & & & 0.034 & -0.147 & 0.903 \\
\hline AVERAGE & 0.038 & -0.144 & 0.806 & 0.058 & 0.060 & 0.076 & 0.038 & -0.110 & 0.878 \\
\hline
\end{tabular}




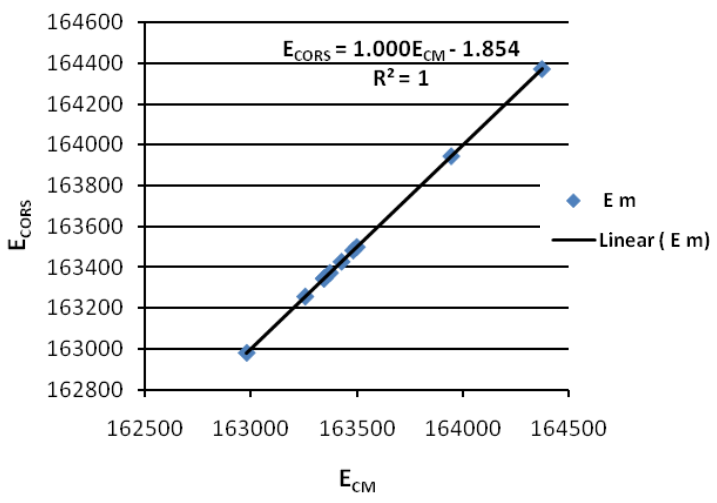

Fig. 2 Graph of the Eastings of points by the Conventional Method versus those of CORS

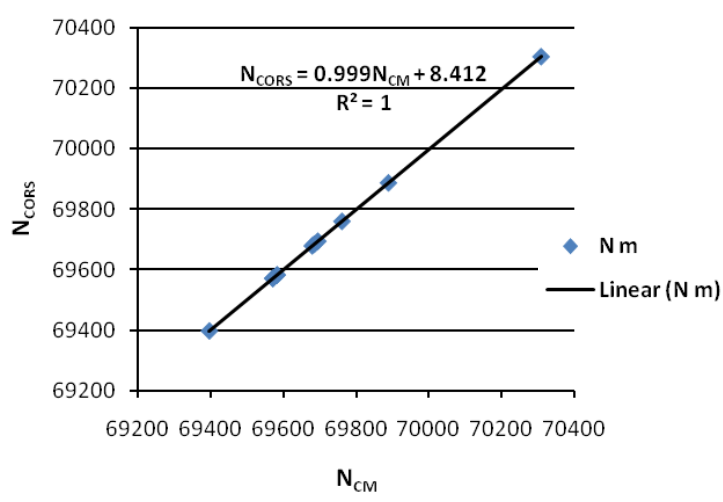

Fig. 3 Graph of the Northings of Points by the Conventional Method versus those of CORS

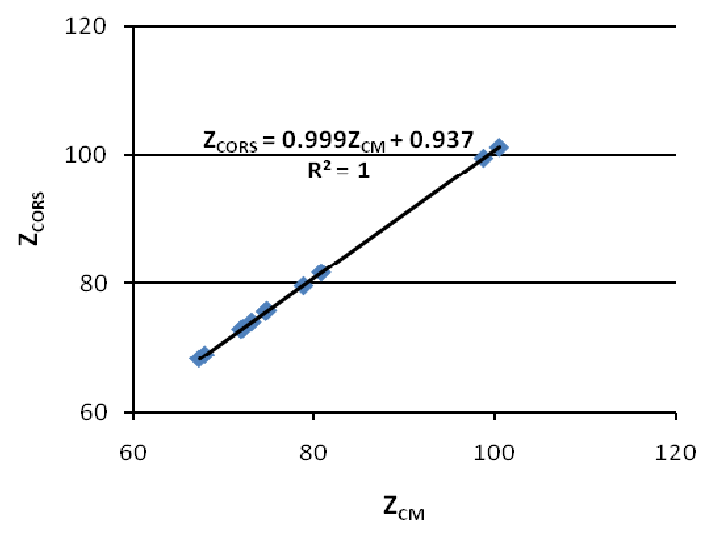

Fig. 4 Graph of the Elevations of Points of Con ventional Method versus those of CORS

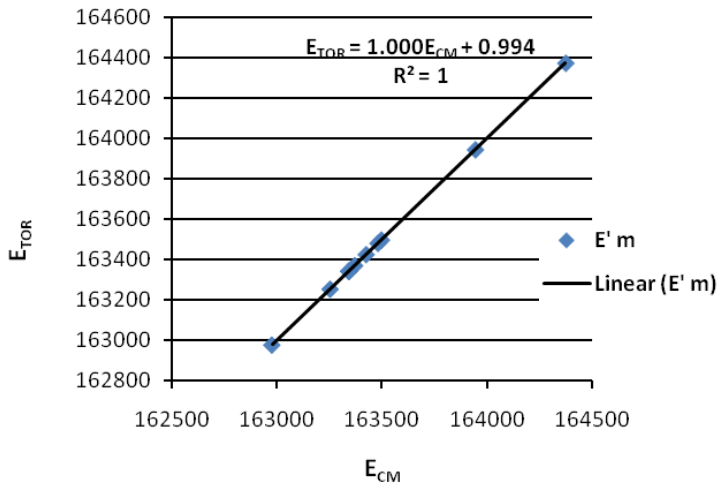

Fig. 5 Graph of the Eastings using the Conventional Method versus those of the Temporary Occupied Reference Station

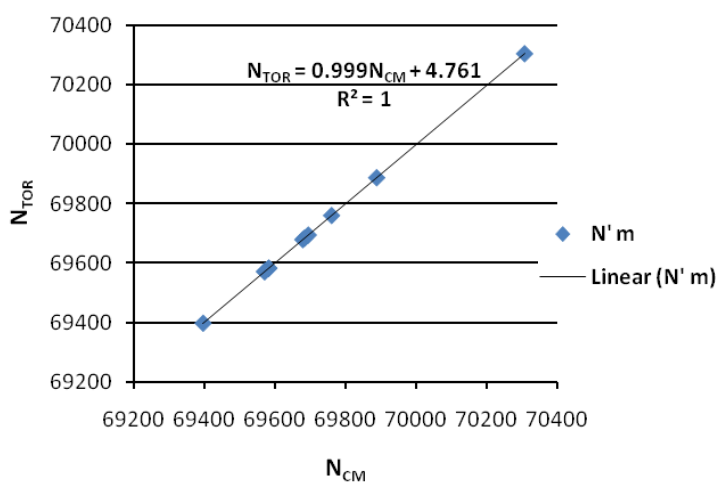

Fig. 6 Graph of the Northings using the Conventional Method versus those of the Temporary Occupied Reference Station

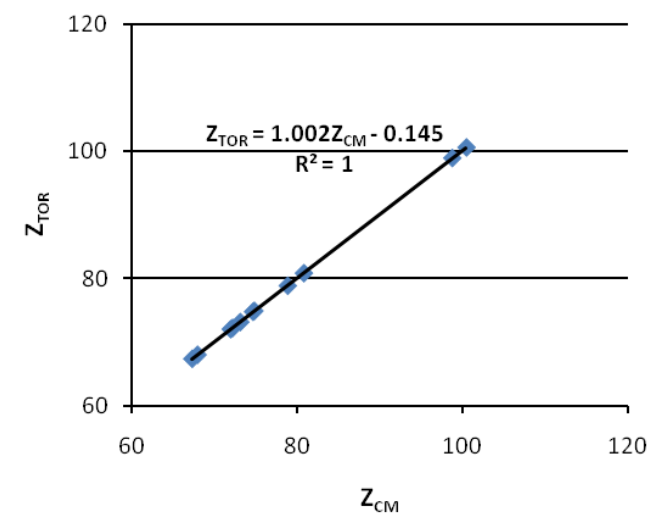

Fig. 7 Graph of the Elevations using the Con ventional Method versus those of the Temporary Occupied Reference Station 
The same pattern was observed in all the three sets of data collected and post-processed in the study. The variations in the easting coordinates in all three methods fell within the acceptable ranges of the accuracies of the instruments used. Errors in the planimetric positions might be due to an inherent error in the reference station. The apparently inherent error in the nothings of the reference station will inevitably affect the northings of the rover stations. This calls for further research. A systematic average discrepancy of $0.806 \mathrm{~m}$ in the coordinates of the heights may be attributed to the fact that the exact value for the geoidal ellipsoid separation has not been determined for the country, and was therefore assigned the value zero during the processing.

\section{Conclusions}

The three techniques employed in the determination of the coordinates of the selected points on the UMaT campus yielded acceptable results consistent with the discrepancies in the GPS postprocessed coordinates of positions fixed with respect to the temporary reference station and those of the CORS.

The results of this work show that the correlation coefficients (r) are equal to +1 thus the scatter plots of the various coordinates of the control points are positively correlated and the regression equations for the eastings, northings and elevation were linear. Thus the CORS located at UMaT Campus can be effectively used in GPS surveys to yield accurate results which agree quite well with the local triangulation network in the region.

\section{References}

Anon. (2004), "CORS Station in St. Clair County", http://www.staircounty.org/officers/cors, Accessed: March 19, 2009.

Anon. (2008), "Testing and Evaluation of a GPS CORS Network for Real Time Centimetric Positioning -The Victoria GPSnet ${ }^{\mathrm{TM}}$ ", http:// www.dpi.vic.gov.au/ ca256f310024b628/ 0/ b0cd755ba17 6edb cca 2571f500271c61/ \$file/ 4019_ignss2006_gordini.pdf, Accessed: July 20, 2008 .

Anon. (2009), "Dupage County Geodetic Survey Control", www.dupageco.org/gis/gps. Accessed: March 19, 2009.

Kesse, G. O. (1985), The Mineral and Rock Resources of Ghana, A. A. Balkema, Rotterdam, $610 \mathrm{pp}$.

Torge, W. (2001), Geodesy, Walter de Gruyter, Berlin, 403 pp.

\section{Authors}

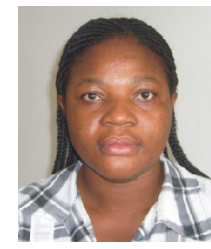

C.B. Boye (Mrs.) holds a Masters degree in GeoInformatics from the International Institute of Geo Information and Earth sciences (ITC), Enschede, Netherlands, and a BSc degree in Geodetic Engineering from the Kwame Nkrumah University of Science and Technology, Kumasi, Ghana. She worked with Kasap Ltd as a geodetic engineer on various road and building projects. In 2004 she joined the University of Mines and technology (UMaT) Tarkwa where she lectures in Engineering Surveying, Cadastral Surveying, Photogrammetry and Remote Sensing. She a member of the Ghana Institution of Engineers, the African Association of Remote Sensing and Environment (AARSE), and an academic member of the Federation of International Surveyors (FIG).

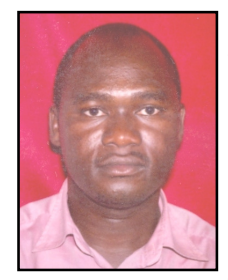

Issaka Yakubu is an Assistant Lecturer at the University of Mines and Technology (UMaT), Tarkwa-Ghana. He holds a Bachelor of Science degree in Geomatic Engineering from the Kwame Nkrumah University of Science and Technology, Kumasi-Ghana. He worked in the Department of Geomatic Engineering of UMaT as a Teaching Assistant from 2005 to 2006. He obtained his Master of Philosophy (MPhil.) degree in Geomatic Engineering from the University of Mines and Technology, Tarkwa-Ghana. He has skills in the usage of ArcGIS 9.x, ILWIS, Spectrum Survey, GNSS Solutions, SurvCAD, AutoCAD and SURPAC software. His research interest includes application of GIS, GPS and Cadastral Surveying. 